\title{
Human papilloma virus (HPV) prevalence upon HPV vaccination in Swedish youth: a review based on our findings 2008-2018, and perspectives on cancer prevention
}

\author{
Juan $\mathrm{Du}^{1}{ }^{1} \cdot$ Andreas Ährlund-Richter ${ }^{2} \cdot$ Anders Näsman $^{2} \cdot$ Tina Dalianis $^{2}$ \\ Received: 28 September 2020 / Accepted: 4 November 2020 / Published online: 27 November 2020 \\ (c) The Author(s) 2020
}

\begin{abstract}
Purpose Three human papillomavirus (HPV) vaccines are available against up to nine HPV types. In Sweden, from 2012, Gardasil was offered to 10-12 year old girls through the school-based vaccination program, and as catchup vaccination for women up to 26 years. To obtain a baseline, and follow HPV vaccination effects, during 2008-2018, cervical and oral HPV prevalence were followed at a youth clinic in Stockholm, and in 2013 for comparison oral HPV prevalence was examined in high-school youth in a middle-sized county in Sweden.

Methods In this review, we discuss all our data with cervical and oral mouthwash samples that were collected and tested for 24-27 HPV types by a bead-based multiplex assay from 2008.

Results Compared with 2008-2011, with 35\% HPV16 and > 60\% high risk (HR) HPV cervical prevalence at the youth clinic, a decrease of vaccine HPV types was observed between 2013 and 2018, with e.g., HPV16 falling to 5\% in catchup vaccinated women and 15-18\% in nonvaccinated women. Most common cervical HR-HPV types were HPV39, 51, 52, 56 , and 59 together accounting for $\sim 10 \%$ of cervical cancer, and where only HPV52 is included in Gardasil-9. At baseline 2009-2011, oral HPV prevalence was $\sim 10 \%$ in unvaccinated youth at the youth clinic, but after 2013 it dropped to $<2 \%$ at the youth clinic and high schools.

Conclusion To conclude, Gardasil HPV types have decreased, but it is still important to follow remaining HR-HPV types and cancer development, since there is an ongoing increase in the incidence of HPV-associated tonsillar and base of tongue cancer, and cervical cancer in Sweden.
\end{abstract}

Keywords HPV prevalence $\cdot$ HPV vaccines $\cdot$ Cervical cancer $\cdot$ Tonsillar cancer $\cdot$ Base of tongue cancer $\cdot$ Screening

\section{Introduction and historic perspective}

In 1983, when HPV16 was finally disclosed in cervical cancer, this finding was complex since several HPV types were being discovered and found to cause cervical cancer [1-5].

Juan Du

juan.du@ki.se

$\bowtie$ Tina Dalianis

Tina.Dalianis@ki.se

1 Department of Microbiology, Tumor and Cell Biology, Centre for Translational Microbiome Research (CTMR), Karolinska Institutet, Stockholm, Sweden

2 Department of Oncology-Pathology, Karolinska Institutet, Stockholm, Sweden
Today, $>200$ HPV types have been discovered, of which most are cutaneous and 40 mucosal and defined as low risk (LR), putatively high risk (HR), or HR-HPV types [5]. All have an $\sim 8 \mathrm{~kb}$ double-stranded circular DNA genome encoding the E1-E2, E4-E7 regulatory proteins. E6 and E7 of HR-HPV types, binding p53 and retinoblastomprotein $(\mathrm{Rb})$ and dysregulating the cell proliferation, are classified as oncogenes [5]. L1 and L2 are structural proteins, form the viral capsid, and L1 capable of forming virus like particles (VLPs) is the basis for HPV vaccines [5].

In 1995, HPV16 was recognized as a carcinogen in cervical cancer by the International Agency for Research against cancer (IARC) [6]. Oropharyngeal squamous cell carcinoma (OPSCC), and its subsites tonsillar and base of tongue cancer (TSCC/BOTSCC) were reported to be associated with HPV 16 infection in 2000 and 2007, respectively. This was also recognized by the IARC [8-10] By then, TSCC and 
BOTSCC were increasing drastically in many Western countries, as has increased in Sweden [11, 12].

In parallel, in 2006, the first HPV vaccine Gardasil, which against HPV16, 18, 6 and 11, was approved by the Food and Drug Administration. This was the case for Cervarix (against HPV16 and 18) and Gardasil-9 (against HPV16, 18, 31, 33, $45,52,58,6$ and 11) in 2009 and 2014, respectively [7].

This review presents details of the period during the introduction of the HPV vaccine with focus on young individuals at a youth clinic in Stockholm, Sweden, but also in youth at high schools in a median-sized county in Sweden (Table 1). In addition, parallel studies on possible interplay of vaginal microbiota and HPV infection, and perspectives on precancerous lesions and screening are briefly commented on.

\section{$H P V$ vaccination in Sweden and background to our studies}

HPV vaccination was gradually introduced in Sweden between 2006-2011. In 2012, Gardasil was supplied for 10-12 year old girls through the school-based vaccination program, and catchup vaccination of girls aged 13-26 years was also offered for free, depending on Swedish county [13]. From September 2019, Gardasil-9 has been used in the school-based vaccination program, and this autumn 2020, boys 10-12 years of age start to be vaccinated in Sweden.

Prior to the introduction of HPV vaccination, we had described HPV causative of TSCC/BOTSCC and reported increasing incidences of HPV-positive TSCC and wanted to follow vaccination effects on oral HPV prevalence [11]. Therefore, we collaborated with the Swedish Agency for Infectious Disease Control and examined cervical and oral HPV prevalence in youth aged 15-23 years at a large youth clinic in Stockholm, Sweden. Investigations conducted during 2008-2010 and 2009-2011 obtained a baseline for cervical and oral HPV prevalence (later found $\sim 70 \%$ and $\sim 10 \%$ respectively) before HPV vaccination and compared HPV types at the two locations [14, 15]. The latter was of interest, due to that e.g., HPV 16 accounted for $\sim 90 \%$ of all HPV positive TSCC/BOTSCC as compared to $\sim 55 \%$ of all cervical cancer $[11,16]$.

In 2013, with colleagues in Uppsala, we performed a study on life style and oral HPV prevalence, in high school

Table 1 Overview of the studies included in this review

\begin{tabular}{|c|c|c|c|c|c|c|c|}
\hline Collection period & Place & Sample size ${ }^{a}$ & Vaccine $\%^{\mathrm{b}}$ & Sample type & HPV\% & Top five HR-HPV types ${ }^{c}$ & $\begin{array}{l}\text { Reference } \\
\text { number }\end{array}$ \\
\hline $2008-2010$ & Youth clinic & 544 & 0 & Cervical samples & $70 \%$ & $16,51,18,52,73$ & 14 \\
\hline 2009-2011 & Youth clinic & 483 & 0 & Oral samples & $9.3 \%$ & $16,59,51,(56,82)$ & 15 \\
\hline 2009-2011 & Youth clinic & 174 & 0 & Cervical samples & $74 \%$ & $16,52,51,18,(56,59)$ & 15 \\
\hline 2013 & High school & 335 & $\begin{array}{l}64 \% \text { in } 160 \\
\text { females }\end{array}$ & Oral samples & $1.8 \%$ & $16,(56,68)$ & 17 \\
\hline $2013-2014$ & Youth clinic & 211 & $73 \%^{\mathrm{a}}$ & Cervical samples & $\begin{array}{l}61 \% \text { in vaccinated \& } \\
70 \% \text { in nonvacci- } \\
\text { nated women }\end{array}$ & $\begin{array}{l}51,(56,59,73), \\
39 \text { in vaccinated women } \\
\& 16,(51,56,39),(59, \\
52,45,31) \text { in nonvac- } \\
\text { cinated women }\end{array}$ & 18 \\
\hline $2013-2014$ & Youth clinic & 287 & $\begin{array}{l}73 \% \text { in } 200 \\
\text { females }\end{array}$ & Oral samples & $1.4 \%$ & $16,(51,52,59)$ & 18 \\
\hline $2013-2015$ & Youth clinic & 338 & $71 \%$ & Cervical samples & $\begin{array}{l}65 \% \text { in vaccinated \& } \\
75 \% \text { in nonvacci- } \\
\text { nated women }\end{array}$ & $\begin{array}{l}56,(59,51),(52,39,73) \\
\text { in vaccinated women } \\
\& 59,16,52,(51,73, \\
82) \text { in nonvaccinated } \\
\text { women }\end{array}$ & 19 \\
\hline $2013-2015$ & Youth clinic & 457 & $\begin{array}{l}71 \% \text { in } 335 \\
\text { females }\end{array}$ & Oral samples & $1.5 \%$ & $\begin{array}{l}(16,51,52,59,39,42 \\
35)\end{array}$ & 19 \\
\hline $2017-2018$ & Youth clinic & 172 & $82 \%$ & Cervical samples & $\begin{array}{l}67 \% \text { in vaccinated \& } \\
93 \% \text { in nonvacci- } \\
\text { nated women }\end{array}$ & $\begin{array}{l}56,59,51,52,39, \text { in } \\
\text { vaccinated women \& } \\
(56,51),(52,39),(73, \\
33,16,45) \text { in nonvac- } \\
\text { cinated women }\end{array}$ & 20 \\
\hline
\end{tabular}

${ }^{\text {a }}$ Samples with enough material; Oral samples were collected from both genders

${ }^{\mathrm{b}}$ Most of the women got catchup vaccination

${ }^{\mathrm{c}}$ More HPV types are listed in () if the prevelence is same 
classes in a middle-sized county in Sweden, and reported that oral HPV prevalence was low, but by then almost $70 \%$ of the young girls had been catchup HPV vaccinated [17]. This prompted us to do follow-up studies 2013-2018 at the youth clinic in Stockholm where changes in cervical and oral HPV prevalence were observed, with decreases in HPV vaccine types, but persistence of HPV types not covered by the vaccines [18-20].

\section{The youth clinic, high schools, the study designs, sample collection, and HPV typing}

\section{The youth clinic}

The first two studies partly overlapped and were conducted 2008-2010 and 2009-2011 in one of the largest youth clinics in Stockholm, visited annually by 4000 women and 800 men aged 15-23 years, for advice on birth control and treatment of sexually transmitted diseases $[14,15]$. The three follow-up studies were performed 2013-2018 [18-20]. The youth were from the local area and the universities of Stockholm. Participation was voluntary, anonymous, and performed after ethics approval. The data on birth year and HPV vaccination status were acquired for all, and for microbiota studies, information on antibiotic treatment was added. In the two first studies, the basis of HPV prevalence data was analysed on only unvaccinated individuals, and the proportion of HPV-catch up vaccinated individuals was $~ 10-20 \%$ $[14,15]$. In the follow up studies, HPV prevalence data was acquired from both HPV nonvaccinated and catchup vaccinated individuals, and by then $\sim 70 \%$ were catchup vaccinated, and with time subsequently some were vaccinated through the school-based vaccination program [18-20]. The participation rate was $\sim 15 \%$ due to the work load of the midwives, who could inform the youth and collect samples maximum 1-2 times per week [14]. Roughly 1200 youth have participated in our studies over the years [14, 15, 18-20].

\section{High schools}

Youth from their first year (877 students) at 13 senior high schools from one municipality with a population of 140,000 in mid-south Sweden, initially participated in a study on health, life style, sexual experiences, attitudes towards sexuality, and pornography consumption. During their third year, a follow up study was conducted, with 627 participants contributed a voluntary collection of mouthwash samples. Among those, 335 students (160 females and 175 males), aged 17-21 years (median age 18 years) donated oral samples for our study, with collection initiated in January 2013 and completed in March 2013 [17].

\section{Sample collections}

Oral samples were obtained by a gargle for $30 \mathrm{~s}$ with $15 \mathrm{ml}$ $50 \%$ Listerine (Johnson and Johnson Consumer Nordic) in plastic containers (Sarstedt, 25-ml screw cap yellow, Sweden), stored at $+4{ }^{\circ} \mathrm{C}$ for $0-3$ days, transported back to the lab and treated as described before [15, 17]. Cervical samples were collected either by a midwife or by self-collectiom using nylon flocked swabs (Copan, Brescia, Italy), and preserved in sterile tubes containing $5 \mathrm{ml}$ SurePath preservation solution (BD, Sweden) or $0.8 \mathrm{ml}$ DNA/RNA shield (Zymo Research, CA, USA), and stored at $+4{ }^{\circ} \mathrm{C}$ before treatment [14].

\section{HPV testing}

Samples were initially tested for 24 and later 27 mucosal HPV types using L1 primers. Extra E6 primers for HPV16 and HPV33, and $\beta$-globin gene primers as controls were also analysed by a Luminex-based multiplex assay, as described before [14-17]. HR-HPV or LR-HPV types were classified according to Muñoz et al., 2006 [21]. Median fluorescence intensity (MFI) $>30$ for $\beta$-globin was defined as adequate DNA quality and data interpretation. Samples with MFIs $>8+1.5 \times$ background were considered HPV positive [14].

\section{Initial HPV prevalence studies 2008-2011, at a large youth clinic in Stockholm, Sweden}

\section{The two initial studies at the youth clinic}

The 1st study (2008-2010) initially included 544 nonvaccinated women donating cervical samples, while the 2 nd study (2009-2011) included 408 women and 82 men donating oral samples, where 180 of the women had also donated cervical samples $[14,15]$. During this period $\sim 10 \%$ of the women had been HPV catchup vaccinated and samples from these women were not included in the analysis [14] (Table 1).

\section{Cervical prevalence}

Cervical HPV prevalence in young women was similar in the 1 st and 2 nd studies, i.e., 70 and $74 \%$, respectively, of the samples tested positive for one or more HPV types, of which most were HR types ( $>60 \%)$, with HPV16 dominating, with $34.7 \%$ and $37.9 \%$, respectively $[14,15]$ (Table 1 ). Other common HR types were HPV52, 51 and 18 at roughly $10-15 \%$. The most common LR type was HPV42 ( 17\%), while LR HPV6 and 11 included in the vaccines were less common (around 8 and $2 \%$, respectively). The highest cervical HPV prevalence was in women aged 19-21 years. 


\section{Oral prevalence}

Oral HPV prevalence in young men and women was similar with $9.3 \%$ for the whole group, $9.8 \%$ in men and $9.2 \%$ in women (Table 1). HR-HPV types accounted for $7.2 \%$ of all oral samples, and HPV16 dominated with 2.9\%, while HPV18 was only detected in one sample $(0.2 \%)$ [15]. Notably, oral HPV prevalence was high in women with cervical HPV infection $(17.1 \%)$ as compared to those with no cervical infection $(4.4 \%)(p=0.043)[15]$.

\section{Oral and cervical prevalence showed concordance in the cervix and oral cavity}

In the 2nd study, we observed that MFI signals in the cervix were higher, and many more HPV types were generally found in the cervix as compared to the oral cavity, likely due to that in the latter $0.5-1.5 \mathrm{~L} /$ day of saliva is produced. Notably, all HPV types detected in the oral cavity were also observed in the cervix, but not vice versa [15].

\section{Oral HPV prevalence among high school students at a medium-sized county in Sweden}

The studies conducted in youth at the youth clinic, were suggested not to be representative of youth in general, since the former may have been more sexually active, and many visited for checking for sexually transmitted diseases. When compared with international reports, both cervical and oral HPV prevalence at the youth clinic in Stockholm was relatively high [14, $15,22,23]$. It was therefore valuable to examine HPV prevalence in a high school setting. Colleagues in Uppsala, were conducting a life style study among high school students, so we joined forces after obtaining ethical permission. Mouth wash samples were collected from 160 females and 175 males (335 in total) high-school students, aged 17-21 years (median 18 years of age). According to the questionnaire, $64 \%$ of the females had been catchup vaccinated against HPV at a median age of 16.4 years, while the median age for sexual debut was 15.2 years. Notably, the overall oral HPV prevalence in this setting was $1.8 \%$, with oral HR-HPV prevalence at $3.1 \%$ $(5 / 160)$ in females (all 18 years of age) and $0.6 \%(1 / 175)$ in males (19 years of age) (Table 1). Among the females, $4 / 5$ HPV infections were with HPV16, and one had an HPV58 infection, and the only male had an oral HPV56 infection. Of the girls 4/5 were HPV vaccinated, between 2009 and 2012, but all had been vaccinated after their sexual debut. The oral HPV prevalence of $1.8 \%$ was much lower than that at the youth clinic 2009-2011, where only few individuals had been vaccinated and we only had baseline data [17]. Therefore, we returned to the youth clinic, to examine possible changes in HPV prevalence there.

\section{Continued studies 2013-2018, on HPV prevalence at the Stockholm youth clinic}

Three studies after each other were conducted, 2013-2015, 2013-2016, and 2017-2018. All three documented similar changes, and the HPV catchup vaccination rate was $>70 \%$ [18-20].

\section{Cervical prevalence}

Cervical prevalence was in these three follow-up studies examined in roughly an additional 500 women (Table 1) [18-20]. The prevalence of HPV16 had dropped to virtually 5\% in catchup HPV vaccinated women, and to roughly $18 \%$ in unvaccinated women, as compared to that reported previously (34.1-37.8\%) [14, 15, 18-20]. Likewise, the cervical prevalence of all HPV vaccine types, when grouped together, had decreased considerably in catchup vaccinated young women, as well as to some extent in nonvaccinated women, while nonvaccine HPV types had remained and were increasing relatively (Fig. 1) [20]. It was also of note that in the latter study 2017-2018, that the youngest
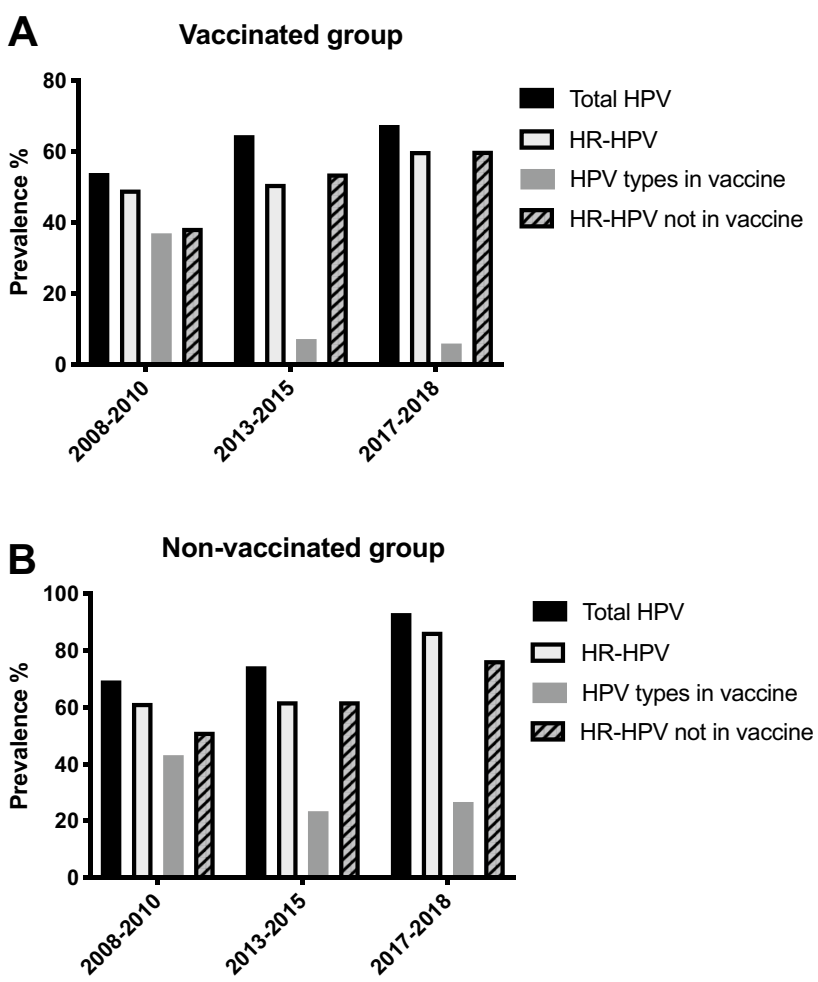

Fig. 1 Cervical HPV prevalence in youth during 2008-2010, 2013-2015, and 2017-2018 at a youth clinic in Stockholm. The data are presented as total HPV prevalence, HR-HPV prevalence, HPV types in the tetravalent vaccine, and HR-HPV types not covered in vaccine among HPV vaccinated (most of the women got catchup vaccination) (a) and non-HPV vaccinated youth (b) 
girls (16-17 years of age) had been vaccinated through the school-based program and they exhibited extremely low prevalence of the HPV vaccine types [20]. Nonetheless, the frequency of HR-HPV infections of which most were not included even in Gardasil-9, remained high and these account for around $10 \%$ of cervical cancer in the same region during the time period 2003-2008 [16, 20].

\section{Oral prevalence}

Oral HPV prevalence also dropped considerably to $1.5 \%$ (7/457) when assessed at the youth clinic in the study of 2013-2015 (Table 1) [19]. Seven samples in total were HPV positive, 3 from men and 4 from women, with only one being positive for HPV 16. Thus, there was a prominent decrease in the oral HPV prevalence in Stockholm as well $[15,17]$.

\section{Cervical and oral HPV prevalence the past 10 years}

To conclude, at the youth clinic both cervical and oral HPV prevalence were fairly high from an international perspective, and in comparison to the relatively few reports that existed during similar periods [16, 17, 24]. However, upon HPV catchup vaccination, HPV prevalence of the HPV vaccine types decreased considerably, although leaving HRHPV types still there [22, 25]. This means that it is important to continue with screening of women for cervical cancer.

\section{Parallel similar studies of the impact of HPV vaccination on cervical HPV prevalence}

In a recent systemic review and meta-analysis, the data from 60 million individuals were covered in 65 articles from 14 high-income countries, and of these 65 articles, 23 followed HPV infection, 29 followed anogenital warts, and 13 followed CIN2 + [26]. The meta-analysis showed compelling evidence of a significant impact of three-dose girls-only HPV vaccination programs, with the tetravalent Gardasil and bivalent Cervarix vaccines, on infections with HPV16 and 18, and HPV31, 33, and 45 as a group. This was also the case for anogenital warts and CIN2 +. The researchers also found evidence of herd immunity. Basically, our data with regard to decreases of the HPV vaccine types in cervical samples of catchup vaccinated women, as well as potential herd immunity in nonvaccinated women are very much in line with the compiled data of the meta-analysis [27]. The data on the effects of HPV vaccines on oral HPV infections are limited. However, a recent European study shows data very similar to ours, with a decrease in oral HPV prevalence after HPV vaccination, as also shown earlier in Costa Rica [27, 28].

\section{Future perspective}

\section{Screening of cervical cancer}

Screening of cervical cancer is according to the National Board of Health and Welfare today recommended, by cytology for women 23-29 years every third year, by HPV testing for women 30-49 years every 3rd year, and by complementary cytology at the age of 41 years [12]. For women 50-64 years of age, HPV testing is done every 7 years. In our opinion, it may be of importance to have both L1 and E6 primers for all HR-HPV types in HPV testing, since it has been documented that L1 can often be lost upon cancer development [5]. Furthermore, importantly, not all HR-HPV types have yet disappeared [20].

\section{Screening for HPV-positive TSCC and BOTSCC}

As mentioned above, TSCC and BOTSCC are increasing in incidence, and possible screening has been discussed. However, the presence of oral HPV infection is relatively rare, likely due to the secretion of saliva in the oral cavity. Furthermore, others and we have shown that the presence of HPV in the oral cavity is not necessarily associated with the presence of HPV in the tonsils, and therefore not necessarily correlated to cancer development [24]. Moreovers, some researchers claimed that there are no pre-stages of OPSCC [25].

Nonetheless, we have shown that patients with HPVpositive TSCC and BOTSCC often have high MFI levels of the tumour HPV type in their oral cavity upon donating a gargle sample, and these levels are roughly 200 times higher than the HPV MFIs in oral rinses of healthy youth $[15,29]$. It was also occasionally possible to disclose cytological changes in oral rinses of TSCC and BOTSCC patients [29]. A very high MFI in a mouth wash could be indicative of a risk for TSCC/BOTSCC; however, it has not been proved as a reliable diagnostic indicator for TSCC/BOTSCC. Likewise, it has been reported that antibodies against E6 can develop $>10$ years before development of an oropharyngeal cancer, but this has not been shown to work on an individual level neither [30].

Notably, recently attempts have also been made to look at pre-stages of TSCC and BOTSCC, and we have also compared dysplastic lesions in TSCC to invasive cancer [31]. Forty genes were shown to be differentially regulated between HPV-positive dysplastic tissue and invasive cancer tissue, and 33 genes from HPV-negativedysplastic 
tissue and invasive cancer tissue. Five of the nine most affected pathways showed similar increased activity in both HPV-positive and negative invasive cancer as compared to dysplastic lesions. Taken together, the data suggest that both HPV-positive and HPV-negative TSCC/ BOTSCC precancerous lesions do exist. Nevertheless, further refinement could be possible, such as using some of the most prominent markers, e.g., SPARC, psoriasin, type I collagen, and galectin-1 in future blood tests for cancer screening [31].

\section{HPV and microbiota}

Vaginal microbiota has recently been linked to an increased risk of sexually transmitted infections, including HPV infection [32]. Our data are also supported by others, showing that vaginal microbiota dominated by non-Lactobacilli species or Lactobacillus iners were associated with three to five times higher odds of any prevalent HPV and two to three times higher for high-risk HPV and dysplasia/cervical cancer compared with Lactobacillus crispatus [33]. Metaanalysis on vaginal microbiota and the risk of HPV and cervical cancer also supported a causal link between vaginal dysbiosis and cervical cancer along with HR-HPV acquisition, persistence, and cervical dysplasia development [34]. A potential relationship of certain microbiota with a faster clearance of HPV infection has been suggested, but this field needs to be investigated further with larger and logituginal corhorts. If this is the case maybe one could abrogate a persistent HPV infection by modifying the vaginal microbiota. This of course would be very valuable information and be very helpful in getting rid of persistent HPV infection.

\section{Concluding remarks}

To summarize, presentation of HPV vaccination in the school-based program and as catchup vaccination has successfully decreased the cervical and oral HPV prevalence of the Gardasil HPV16, 18, 6, and 11 types, both in vaccinated and nonvaccinated youth. Continued HPV vaccination on both genders would be extremely valuable and allow for a further decrease in HPV vaccine types and their associated cancers [35].

Nonetheless, other HR-HPV types still persist, of which most are not included in Gardasil-9 and these accounted for $10 \%$ of all cervical cancer in the Stockholm region 2003-2008. Therefore, it is important to follow-up patients with cervical screening, and investigate which HR-HPVs are present in HPV-associated tumors in the future.

In addition, more knowledge on microbiota may allow for better means of combating persistent HPV infections.
Future cancer screening could also use the knowledge acquired on different protein expression in dysplastic and invasive lesions of TSCC and BOTSCC.

Acknowledgments This work was supported by the Swedish Research Council, the Swedish Cancer Foundation, the Stockholm Cancer Society, the Swedish Cancer and Allergy Foundation, the Stockholm City Council and the Karolinska Institutet, Sweden.

Funding Open access funding provided by Karolinska Institute.

\section{Compliance with ethical standards}

Conflict of interest The authors report no conflicts of interest.

Open Access This article is licensed under a Creative Commons Attribution 4.0 International License, which permits use, sharing, adaptation, distribution and reproduction in any medium or format, as long as you give appropriate credit to the original author(s) and the source, provide a link to the Creative Commons licence, and indicate if changes were made. The images or other third party material in this article are included in the article's Creative Commons licence, unless indicated otherwise in a credit line to the material. If material is not included in the article's Creative Commons licence and your intended use is not permitted by statutory regulation or exceeds the permitted use, you will need to obtain permission directly from the copyright holder. To view a copy of this licence, visit http://creativecommons.org/licenses/by/4.0/.

\section{References}

1. DiMaio D (2015) Nuns, warts, viruses and cancer. Yale J Biol Med 88:127-129

2. Durst M, Gissmann L, Ikenberg H, zur Hausen H (1983) A papillomavirus DNA from a cervical carcinoma and its prevalence in cancer biopsy samples from different geographic regions. Proc Natl Acad Sci USA 80:3812-3815

3. Boshart M, Gissmann L, Ikenberg H, Kleinheinz A, Scheurlen W, zur Hausen H (1984) A new type of papillomavirus DNA, its presence in genital cancer biopsies and in cell lines derived from cervical cancer. Embo J. 3:1151-1157

4. Munoz N, Bosch FX (1992) HPV and cervical neoplasia: review of case-control and cohort studies. IARC Sci Publ 119:251-261

5. Tommasino M (2014) The human papillomavirus family and its role in carcinogenesis. Semin Cancer Biol 26:13-21. https://doi. org/10.1016/j.semcancer.2013.11.002 (Epub 2013 Dec 4)

6. IARC (1995) IARC Monographs on the evaluation of Carcinogenic Risks to Humans, vol 64. Human Papillomaviruses, Lyon

7. Barra F. Maggiore URL, Bogani Get al. New prophylactic human papillomavirus (HPV) vaccines against cervical cancer. J Obstr Gynaecol 2019:39

8. Gillison ML, Koch WM, Capone RB et al (2000) Evidence for a causal association between human papillomavirus and a subset of head and neck cancers. J Natl Cancer Inst 92:709-720

9. Mellin H, Friesland S, Lewensohn R et al (2000) Human papillomavirus (HPV) DNA in tonsillar cancer: clinical correlates, risk of relapse, and survival. Int J Cancer 89:300-304

10. WHO. IARC Monographs on the Evaluation of carcinogenic risk to humans. Lyon, France: International Agency for Research on Cancer; 2007.

11. Ramqvist T, Dalianis T (2010) Oropharyngeal cancer epidemic and human papillomavirus. Emerg Infect Dis 16:1671-1677 
12. https://www.socialstyrelsen.se

13. Vänskä S, Söderlund-Strand A, Uhnoo I et al (2018) Estimating effectiveness of HPV vaccination against HPV infection from post- vaccination data in the absence of baseline data. Vaccine 36:3239-3246

14. Ramqvist T, Du J, Lundén M, Ahrlund-Richter S, Ferreira J, Marions L, Sparén P et al (2011) Pre-vaccination prevalence of human papillomavirus types in the genital tract of 15-23-year-old women attending a youth health clinic in Stockholm. Sweden Scand J Infect Dis 43:115-121

15. Du J, Nordfors C, Ahrlund-Richter A et al (2012) Prevalence of oral human papillomavirus infection among youth. Sweden Emerg Infect Dis 18:1468-1471

16. Du J, Näsman A, Carlson JW et al (2011) Prevalence of human papillomavirus (HPV) types in cervical cancer 2003-2008 in Stockholm, Sweden, before public HPV vaccination. Acta Oncol 50:1215-1219

17. Nordfors C, Grün N, Haeggblom L, Tertipis N et al (2013) Oral human papillomavirus prevalence in high school students of one municipality in Sweden. Scand J Infect Dis 45:878-881

18. Grün N, Ährlund-Richter A, Franzén J, Mirzaie L, Marions L, Ramqvist T, Dalianis T (2015) Oral human papillomavirus (HPV) prevalence in youth and cervical HPV prevalence in women attending a youth clinic in Sweden, a follow up-study 2013-2014 after gradual introduction of public HPV vaccination. Infect Dis (Lond) 47(1):57-61

19. Grün N, Ährlund-Richter A, Franzén J et al (2016) Follow-up on oral and cervical human papillomavirus prevalence 2013-2015 in youth at a youth clinic in Stockholm, Sweden. Infect Dis (Lond) 48:169-170

20. Ährlund-Richter A, Cheng L, Hu YOO, Svensson M, Pennhag AAL, Ursu RG et al (2019) Changes in cervical human papillomavirus (HPV) prevalence at a youth clinic in Stockholm, Sweden, a decade after the introduction of the HPV vaccine. Front Cell Infect Microbiol 9:59

21. Manos MM, Ting Y, Wright DK et al (1989) Use of polymerase chain reaction amplification for the detection of genital human papillomaviruses. Cancer Cells 7:209-214

22. Clifford GM, Gallus S, Herrero R et al (2005) Worldwide distribution of human papillomavirus types in cytologically normal women in the International Agency for Research on Cancer HPV prevalence surveys: a pooled analysis. Lancet 366:991-998

23. Drolet M, Bénard É, Pérez N, Brisson M; HPV Vaccination Impact Study Group. Population-level impact and herd effects following the introduction of human papillomavirus vaccination programmes: updated systematic review and meta-analysis. Lancet. 2019;394:497-509.

24. Grün N, Mbuya W, Ternhag A et al (2017) Human papillomavirus prevalence in mouthwashes of patients undergoing tonsillectomy shows dominance of HPV69, without the corresponding finding in the tonsils. Infect Dis (Lond) 49:588-593
25. Holmwa BJ, Wenig BM (2019) Virus-associated carcinomas of the head \& neck: Update from the 2017 WHO classification. Ann Diagn Pathol. 38:29-42

26. Mélanie D, Élodie B, Norma P, Marc B, the HPV Vaccination Impact Study Group. Population-level impact and herd effects following the introduction of human papillomavirus vaccination programmes: updated systematic review and meta-analysis. Lancet. 2019;394: 497-509.

27. Malerova S, Hejtmankova A, Hamsikova E, Salakova M, Smahelova J, Klozar J, Tachezy R (2020) Prevalence and risk factors for oral HPV in healthy population, in Central Europe. Anticancer Res 40:1597-1604

28. Herrero R, Quint W, Hildesheim A, Gonzalez P, Struijk L, Katki HA, Porras C, Schiffman M, Rodriguez AC, Solomon D, Jimenez S, Schiller JT, Lowy DR, van Doorn LJ, Wacholder S, Kreimer AR; CVT Vaccine Group. Reduced prevalence of oral human papillomavirus (HPV) 4 years after bivalent HPV vaccination in a randomized clinical trial in Costa Rica. 2013;8(7):e68329.

29. Nordfors C, Vlastos A, Du J et al (2014) Human papillomavirus prevalence is high in oral samples of patients with tonsillar and base of tongue cancer. Oral Oncol 50:491-497

30. Kreimer AR, Shiels MS, Fakhry C et al (2018) Screening for human papillomavirus-driven oropharyngeal cancer: considerations for feasibility and strategies for research. Cancer 124:1859-1866

31. Haeggblom L, Ährlund-Richter A, Mirzaie L et al (2019) Differences in gene expression between high-grade dysplasia and invasive $\mathrm{HPV}^{+}$and $\mathrm{HPV}^{-}$tonsillar and base of tongue cancer. Cancer Med 8:6221-6232

32. Cheng LQ, Norenhag J, Hu YOO et al (2020) Vaginal microbiota and human papillomavirus infection among young Swedish women. NPJ Biofilms and Microbiomes 6(1):1-10

33. Norenhag J, Du J, Olovsson M, Verstraelen H et al (2020) The vaginal microbiota, human papillomavirus and cervical dysplasia: a systematic review and network meta-analysis. BJOG 127:171-180

34. Brusselaers N, Shrestha S, van de Wijgert J, Verstraelen H (2019) Vaginal dyspoiosis and the risk of human papillomavirus and cervical cancer: systematic review and meta-analysis. Am J Obstdg Gynecol 221:9-18

35. Näsman A, Du J, Dalianis T (2020) A global epidemic increase of an HPV-induced tonsil and tongue base cancer-potential benefit from a pan-gender use of HPV vaccine. J Intern Med 287:134-152

Publisher's Note Springer Nature remains neutral with regard to jurisdictional claims in published maps and institutional affiliations. 